http://dx.doi.org/10.1590/1678-4162-8735

Arq. Bras. Med. Vet. Zootec., v.68, n.4, p.1023-1032, 2016

\title{
Exigências de energia de borregas mestiças alimentadas com níveis crescentes de concentrado na dieta
}

\author{
[Energy requirements of crossbreed ewe lambs fed with increasing dietary \\ levels of concentrate] \\ M.G. Morais ${ }^{1}$, H.J. Fernandes ${ }^{2}$, L.B. Oliveira ${ }^{1}$, C.B. Ribeiro ${ }^{1}$, A.R.D.L. Souza ${ }^{1 *}$, G.L. Franco ${ }^{1}$, \\ C.C.B.F. Ítavo ${ }^{1}$, A.G.F. Andrade ${ }^{1}$, L.C.V. Itavo ${ }^{1}$, G.K.A. Melo ${ }^{1}$, C.C. Walker ${ }^{1}$ \\ ${ }^{1}$ Universidade Federal de Mato Grosso do Sul - Campo Grande, MS \\ ${ }^{2}$ Universidade Estadual de Mato Grosso do Sul - Aquidauana, MS
}

\begin{abstract}
RESUMO
Objetivou-se estimar as exigências energéticas de borregas mestiças confinadas, alimentadas com níveis crescentes de concentrado na dieta. Foram avaliadas 36 borregas, com peso corporal inicial de $23,7 \pm 3,67 \mathrm{~kg}$. Seis animais foram abatidos no início do experimento para obtenção dos valores do grupo referência. Os demais (30 animais) foram distribuídos em cinco tratamentos: mantença (alimentação restrita com feno de capim Tifton) e suplementados com níveis crescentes de concentrado (20, 40,60 e 80\%) em base de matéria seca (seis animais por tratamento). As borregas foram abatidas quando atingiram $37,70 \pm 9,89 \mathrm{~kg}$. Os níveis de concentrado influenciaram a eficiência de utilização da energia metabolizável para mantença $(\mathrm{km})$ e consequentemente as exigências diárias de energia metabolizável para mantença. A exigência de energia líquida para mantença de borregas mestiças em crescimento é $57 \mathrm{kcal} / \mathrm{PCJ}^{0,75} / \mathrm{dia}$, em que PCJ é o peso corporal em jejum. As borregas com $20 \mathrm{~kg}$ apresentaram exigência de energia líquida para $100 \mathrm{~g}$ de ganho de peso diário de $465 \mathrm{kcal} / \mathrm{dia}$. As borregas com $40 \mathrm{~kg}$ de peso corporal exigiram $930 \mathrm{kcal} /$ dia de energia líquida para o mesmo ganho. A exigência líquida para mantença de borregas pode ser estimada pela equação: ELm (Mcal/dia) $=$ $0,057 * \mathrm{PCJ}^{0,75}$. A exigência líquida de energia para ganho de borregas mestiças (Mcal/dia) pode ser estimada a partir da equação: $E L g=0,524 \times$ PVJ $^{0,75} \times$ GPCVZ $^{1,21}$, em que GPCVZ é o ganho de peso do corpo vazio. As dietas influenciam as eficiências de usos da energia metabolizável para mantença $(\mathrm{km})$ e ganho $(\mathrm{kg})$.
\end{abstract}

Palavras-chave: ovino, concentrado, energia metabolizável, energia retida

\begin{abstract}
The aim of this study was to estimate the energy requirements of crossbreed ewe lambs in a feedlot fed with increasing levels of concentrate in the diet. 36 ewe lambs were evaluated with initial body weight of $23.7 \pm 3.67 \mathrm{~kg}$. Six animals were slaughtered at the beginning of the experiment to obtain the reference group values. Animals (30) were distributed in five treatments: maintenance (feed restricted with Tifton grass hay) and those supplemented with increasing dietary levels of concentrate (20,40, 60 and 80\%) on a dry matter basis (six animals per treatment). The ewe lambs were slaughtered when they reached $37.70 \pm 9.89 \mathrm{~kg}$. The concentrate levels influenced the utilization efficiency of metabolizable energy for maintenance ( $\mathrm{km}$ ) and consequently the daily requirements of metabolizable energy for maintenance. The net energy requirements for maintenance of crossbred lambs in growth is $57 \mathrm{kcal} / \mathrm{FCW} \mathrm{W}^{0.75} /$ day, where FCW is body weight on fasting. The ewe lambs with $20 \mathrm{~kg}$ presented net energy requirement for $100 \mathrm{~g}$ daily weight gain of $465 \mathrm{kcal} /$ day. The ewe lambs with $40 \mathrm{~kg}$ of body weight required $930 \mathrm{kcal} /$ day of net energy for the same gain. The net energy requirement for maintenance of ewe lambs can be estimated by the equation: $\mathrm{NEm}$ (Mcal/day) $=0.057 *$ $F C W^{0.75}$. The net energy requirement for crossbred ewe lambs gain (Mcal/day) can be estimated from the

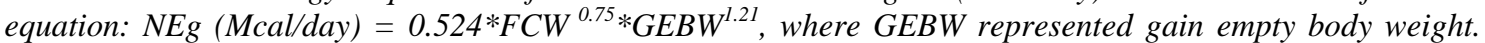
Diets affect the efficiencies of uses of metabolizable energy for maintenance $(\mathrm{km})$ and gain $(\mathrm{kg})$.
\end{abstract}

Keywords: sheep, concentrate, metabolizable energy, retained energy

Recebido em 8 de setembro de 2015

Aceito em 11 de fevereiro de 2016

* Autor para correspondência (corresponding author)

E-mail: andreardl_dagher@yahoo.com.br 


\section{INTRODUÇÃO}

As informações sobre exigências nutricionais e composição dos alimentos representam a alternativa mais eficaz para aumentar produtividade e reduzir os custos das dietas de ovinos criados no Brasil, pois a intensificação dos sistemas, a melhoria da qualidade da carcaça e a redução do ciclo de produção necessitam dessas informações para o adequado balanceamento de dietas e obtenção do máximo desempenho.

A energia disponível dos alimentos é utilizada pelos seres vivos após a oxidação dos nutrientes absorvidos, que gera energia livre para manter as funções biológicas das células dos animais. $\mathrm{O}$ requerimento energético de mantença é definido como a energia necessária para manter os processos biológicos normais, como termorregulação, circulação, respiração e digestão dos animais. Já o requerimento energético para ganho é definido como a energia retida no tecido depositado no ganho de peso, relacionado diretamente com a composição química do corpo vazio (Nutrient..., 2007).

As variações nas exigências energéticas observadas entre os ovinos produzidos no Brasil são decorrentes das diferenças entre raças, das condições fisiológicas, da idade, do sexo, da atividade física e da temperatura ambiental. As divergências entre esses fatores e a falta de adequação das recomendações dos comitês internacionais para as condições de produção brasileira podem comprometer o desempenho animal se somente a base de dados internacionais sobre exigências nutricionais, como Nutrient... (2007), for considerada.

Assim, a condução de pesquisas para obtenção de resultados nacionais sobre as exigências nutricionais de ovinos, especialmente de borregas, é fundamental devido à escassez de informações na literatura. $\mathrm{O}$ conhecimento obtido pode contribuir para melhoria ou validação das recomendações de comitês internacionais, o que tornaria sua utilização mais consistente na formulação de dietas para ovinos no Brasil.
Objetivou-se estimar as exigências energéticas de borregas mestiças confinadas, alimentadas com níveis crescentes de concentrado na dieta.

\section{MATERIAL E MÉTODOS}

O experimento foi conduzido na Faculdade de Medicina Veterinária e Zootecnia da Universidade Federal de Mato Grosso do Sul, em Campo Grande/MS, no período de agosto a dezembro. Foram utilizadas 36 borregas lanadas (1/2 Pantaneira $+1 / 2$ Texel), com peso corporal inicial de $23,7 \pm 3,67 \mathrm{~kg}$ e $4 \pm 1$ meses de idade. O experimento foi aprovado pela Comissão de Ética no Uso de Animais da UFMS (processo $\mathrm{n}^{\mathrm{o}}$ 235/2009).

No início do experimento, os animais foram pesados e distribuídos aleatoriamente em seis grupos com seis animais em cada. Após 28 dias de adaptação, o primeiro grupo foi abatido para determinação da composição corporal inicial dos animais. $\mathrm{O}$ segundo grupo de animais permaneceu durante o período experimental em mantença, com alimentação restrita de feno de capim Tifton 85 (Cynodon spp.). Os demais grupos receberam dietas compostas com feno de capim Tifton 85 (Cynodon spp.) e quatro níveis de concentrado à base de milho, farelo de soja e ureia $(20,40,60$ e $80 \%)$.

As dietas (Tab. 1) foram formuladas de acordo com o Nutrient... (2007), para ganhos de peso de $100,150,200$ e $250 \mathrm{~g} /$ dia, respectivamente.

A água e o suplemento mineral foram fornecidos ad libitum durante todo o período experimental. As pesagens dos animais foram realizadas no início do período experimental e a cada 28 dias para monitoramento do peso corporal em jejum (PCJ). Os animais foram submetidos a 16 horas de jejum prévio de sólidos e pesados imediatamente antes do abate para a obtenção do peso corporal ao abate (PCA). O abate de todos os grupos, inclusive dos animais do tratamento mantença, foi realizado quando os animais do tratamento com $80 \%$ de concentrado atingiram, em média, 48kg de PCJ. Ao fim do período experimental, os animais suplementados com níveis crescentes de concentrado apresentaram, em média, 41,9kg de PCJ. 
Exigências de energia...

Tabela 1. Composição química dos alimentos e das dietas experimentais, em base de matéria seca

\begin{tabular}{|c|c|c|c|c|}
\hline \multirow{2}{*}{ Componentes $^{1}$} & \multicolumn{4}{|c|}{ Alimentos } \\
\hline & Feno & & Concentrado $^{2}$ & Ureia \\
\hline Matéria seca & 924 & & 904 & 980 \\
\hline Matéria orgânica & 938 & & 941 & - \\
\hline Proteína bruta & 107 & & 253 & 2820 \\
\hline Extrato etéreo & 23,3 & & 28,7 & - \\
\hline Fibra em detergente neutro & 770 & & 304 & - \\
\hline Fibra em detergente ácido & 416 & & 56,5 & - \\
\hline Nitrogênio insolúvel em detergente neutro & 8,7 & & 7,1 & - \\
\hline Nitrogênio insolúvel em detergente ácido & 2,3 & & 1,8 & - \\
\hline Lignina & 52 & & 4,6 & - \\
\hline Carboidratos não fibrosos ${ }^{3}$ & 36,9 & & 355 & - \\
\hline \multirow{2}{*}{ Dietas } & \multicolumn{4}{|c|}{ Níveis de concentrado } \\
\hline & $20 \%$ & $40 \%$ & $60 \%$ & $80 \%$ \\
\hline Matéria seca & 919,8 & 915,8 & 911,8 & 907,8 \\
\hline Matéria orgânica & 938,3 & 938,8 & 939,4 & 939,9 \\
\hline Proteína bruta & 202,3 & 209,2 & 216,1 & 223,1 \\
\hline Extrato etéreo & 24,4 & 25,4 & 26,5 & 27,6 \\
\hline Fibra em detergente neutro & 676,6 & 583,5 & 490,4 & 397,3 \\
\hline Fibra em detergente ácido & 344,0 & 272,1 & 200,2 & 128,4 \\
\hline $\mathrm{N}$ insolúvel em detergente neutro & 8,4 & 8,0 & 7,7 & 7,4 \\
\hline $\mathrm{N}$ insolúvel em detergente ácido & 2,2 & 2,1 & 2,0 & 1,9 \\
\hline Lignina & 42,5 & 33,0 & 23,6 & 14,1 \\
\hline Carboidratos não fibrosos ${ }^{3}$ & 100,6 & 164,3 & 228,0 & 291,7 \\
\hline $\mathrm{NDT}^{4}(\%)$ na recria & 63,94 & 72,30 & 76,76 & 77,42 \\
\hline $\mathrm{NDT}^{4}(\%)$ na terminação & 68,17 & 73,11 & 76,97 & 76,27 \\
\hline
\end{tabular}

${ }^{1}$ Matéria seca expressa em $\mathrm{g} / \mathrm{kg}$ e demais componentes em g/kgMS.

${ }^{2}$ Ingredientes: milho integral moído, farelo de soja, levedura seca de cervejaria, melaço de cana em pó, premix mineral vitamínico, fosfato bicálcico, carbonato de cálcio, bicarbonato de sódio e ureia.

${ }^{3}$ Estimado pela equação proposta por Hall (2000). CNF $=\mathrm{MO}-(\mathrm{PB}+\mathrm{EE}+\mathrm{FDN}-\mathrm{PBureia}+$ Ureia), em que CNF são carboidratos não fibrosos, MO é matéria orgânica, PB é proteína bruta, EE é extrato etéreo e FDN é fibra em detergente neutro.

${ }^{4}$ Valores oriundos de cinco ensaios de digestibilidade aparente, três na fase de recria e dois na fase de terminação (Ribeiro, 2011).

Durante o abate, os animais foram insensibilizados por concussão cerebral, com pistola de dardo cativo. Imediatamente procedeuse ao corte da carótida e da jugular e todo o sangue foi coletado, pesado, amostrado e acondicionado em recipientes para posteriores análises. Após a sangria, efetuou-se a esfola, a evisceração e a separação de cabeça e patas. Foram pesados individualmente os órgãos internos (coração, pulmão/traqueia/ esôfago, língua, baço, fígado/vesícula biliar, rins, sistema reprodutivo, bexiga vazia e úbere), os componentes do trato gastrintestinal (TGI) vazio (rúmen/retículo, omaso, abomaso, intestino delgado e intestino grosso), a gordura renal, a gordura omental/mesentérica, a cabeça, os pés e o couro (pele e lã). O peso de corpo vazio
(PCVZ) foi obtido por meio do somatório do peso de todos os constituintes do corpo do animal. Todos os componentes corporais não pertencentes à carcaça dos animais foram congelados para posteriores análises.

A carcaça de cada animal foi pesada logo após o abate e, em seguida, resfriada em câmara fria a $4^{\circ} \mathrm{C}$ por 24 horas. Decorrido esse tempo, as carcaças foram divididas em duas metades $\mathrm{e}$ pesadas. A meia carcaça esquerda foi totalmente dissecada para pesagem dos componentes (ossos, músculos e gorduras) e determinação da composição física dela. Posteriormente, a gordura e os músculos da carcaça foram moídos, quarteados e congelados em freezer a $-4^{\circ} \mathrm{C}$. 
Os ossos da carcaça foram serrados com uma serra de fita em pequenos pedaços, assim como os tecidos da cabeça e dos pés, obtendo-se uma amostra composta de ossos (carcaça, cabeça e patas). Os órgãos e os componentes do TGI, ainda congelados, foram moídos, homogeneizados e quarteados, obtendo-se uma amostra composta de órgãos e TGI. O couro foi tosquiado e pesado. A lã foi amostrada separadamente da pele. A amostragem da pele foi realizada retirando-se um quadrado de $20 \times 20 \mathrm{~cm}$, sendo $2 / 3$ dessa área retirados da parte dorsal e $1 / 3$ da parte ventral do corpo do animal.

Após os procedimentos de amostragem, as amostras (representativas dos componentes corporais) foram pré-secas em estufa com circulação de ar a $65^{\circ} \mathrm{C}$ por 72 horas, em seguida, desengorduradas por 72 horas com éter de petróleo e, posteriormente, moídas. Todas as amostras foram analisadas quanto aos teores de matéria seca (MS), proteína bruta (PB) e extrato etéreo (EE) de acordo com AOAC (1990). O total de energia presente no corpo dos animais foi calculado considerando-se o total de proteína e lipídeos no corpo e o valor energético desses constituintes corporais (Nutrient..., 1980).

A ingestão diária de EM (IDEM) foi estimada a partir do consumo diário de NDT de cada animal (Ribeiro, 2011), conforme sugerido pelo Nutrient... (2007). A energia retida diariamente no corpo foi calculada como a diferença entre o total de energia presente no corpo dos animais ao abate e no início do experimento (estimada com base no peso vivo de cada animal ao início do experimento e no teor de energia do corpo dos animais referência), dividida pelos dias que cada animal permaneceu no experimento. A produção diária de calor (PC) para cada animal foi estimada pela diferença entre a IDEM e a energia retida diariamente no corpo (ER).

As exigências de energia líquida para mantença (ELm) foram estimadas a partir da regressão entre a PC e a IDEM (ambas expressas em $\mathrm{Mcal} / \mathrm{kg}$ PCJ ${ }^{0,75}$ ). Avaliou-se a utilização de um modelo linear e de um modelo exponencial para descrever a relação entre essas variáveis (Eq. [1] e [2])

$$
\begin{aligned}
& \mathrm{PC}=\mathrm{a}+\mathrm{b} \times \text { IDEM } \\
& \mathrm{PC}=\mathrm{a} \times \mathrm{e}^{(\mathrm{b} \times \text { IDEM })}
\end{aligned}
$$

em que: PC é a produção diária de calor (Mcal / $\left.\mathrm{kg} \quad \mathrm{PV}^{0,75}\right)$; “a" e "b" são parâmetros das equações; "e" é a base do logaritmo natural; e IDEM é a ingestão diária de energia metabolizável (Mcal/kg PV ${ }^{0,75)}$.

As exigências de energia metabolizável para mantença $(\mathrm{EMm})$ foram estimadas por regressão linear entre ER e IDEM (Eq [3]).

$\mathrm{ER}=\mathrm{a}+\mathrm{b} \times$ IDEM $\quad$ Eq. [3],

em que: ER é o conteúdo líquido de energia no ganho de peso (Mcal/kg PCJ $\mathrm{P}^{0,75} / \mathrm{dia}$ ); "a" e "b" são parâmetros da equação; e IDEM é a ingestão diária de energia metabolizável (Mcal $/ \mathrm{kg}$ $\mathrm{PCJ}^{0,75} /$ dia). Nesse caso, quando o valor de ER se igualou a zero, foi possível estimar a EMm como a IDEM naquele ponto.

A eficiência de uso da energia metabolizável para mantença $(\mathrm{km})$ foi estimada como a razão entre as exigências de Elm e EMm.

A energia líquida para ganho (ELg) foi estimada por um modelo que utilizou o peso corporal em jejum (PCJ) e o GPCVZ:

$\mathrm{ELg}=\mathrm{a} \times \mathrm{PCJ}^{0.75} \times \mathrm{GPCVZ}^{\mathrm{b}} \quad$ Eq. [4], em que: ELg é o conteúdo líquido de energia no ganho de peso (Mcal/dia); "a" e "b" são os parâmetros da equação; PCJ é o peso corporal em jejum (kg); e GPCVZ é o ganho de peso de corpo vazio $(\mathrm{kg} / \mathrm{dia})$.

A eficiência de uso da EM para ganho $(\mathrm{kg})$ foi estimada como a inclinação da regressão entre a ER e a IDEM (parâmetro "b", Eq. [3]). As exigências de energia metabolizável para ganho (EMg) foram estimadas pela relação entre as exigências de ELg e kg.

O ajuste de todos os modelos e de estimação dos parâmetros foi realizado utilizando-se os procedimentos (PROC) REG (para os modelos lineares) e NLIN (para os modelos não lineares) do SAS v.9.2 (SAS Inst. Inc., Cary, CA). Os outliers foram testados pela avaliação dos resíduos studentizados em relação aos valores preditos pelo modelo. Os dados foram removidos se os resíduos studentizados estivessem fora da faixa de $-2,5$ a 2,5. Na estimativa dos parâmetros das equações de predição das exigências nutricionais, foram removidos os dados de animais do grupo mantença, pois estes apresentaram GPCVZ negativos, provavelmente devido às diferentes formas de utilização dos nutrientes que foram mobilizados do corpo. 
A avaliação da adequação dos modelos foi realizada como sugerido por Tedeschi (2006), utilizando-se o coeficiente de determinação $\left(R^{2}\right)$, o coeficiente de correlação e concordância (CCC), a raiz quadrada do quadrado médio do erro da predição (RQMEP) e a partição do quadrado médio do erro de predição em vício médio, vício sistemático e erro aleatório. Finalmente, foi utilizada a análise do quadrado médio do erro da predição pareado (Wallach e Goffinet, 1989) para comparação entre os modelos quanto à acurácia das predições e o critério delta de informação de Akaike (Burnham e Anderson, 2002) para comparação entre os modelos quanto à precisão das predições.

Todos os cálculos das estatísticas de avaliação e da comparação de modelos foram realizados utilizando-se o MES - Model Evaluation System (http.//nutritionmodels.tamu.edu/mes.htm,

College Station, TX, USA; Tedeschi, 2006). Os modelos de predição das exigências para cada nível de concentrado foram comparados utilizando-se uma variável Dummy, como sugerido por Regazzi (2003).
Uma vez identificado efeito significativo do nível de concentrado na dieta para qualquer um dos modelos de predição, estudaram-se os efeitos lineares e quadráticos do nível de concentrado na dieta sobre os parâmetros daquele modelo. Quando significativo, ajustaram-se modelos de regressão para predição dos parâmetros das equações a partir do nível de concentrado ofertado. Utilizou-se o PROC REG do software SAS v.9.2 (SAS Inst. Inc., Cary, CA) para ajuste das equações de predição. $\mathrm{O}$ nível de significância de 5\% foi adotado em todas as análises estatísticas.

\section{RESULTADOS E DISCUSSÃO}

Para a regressão da produção de calor (PC) em função da ingestão diária de energia metabolizável (IDEM), foi utilizado um modelo linear (Fig.1), pois, ao compará-lo com modelo exponencial quanto à acurácia e à precisão, observou-se similaridade entre ambos. Assim, o modelo linear foi adotado no presente estudo pela maior simplicidade e facilidade de ajuste.

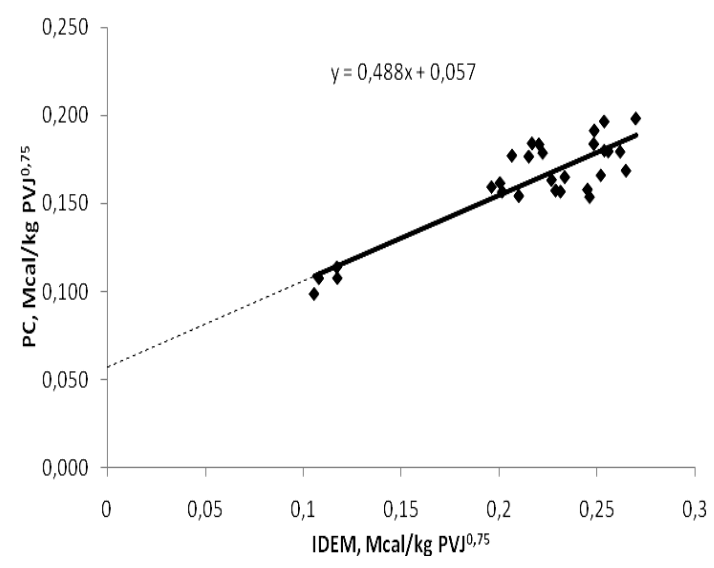

Figura 1. Produção diária de calor (PC) em função da ingestão diária de energia metabolizável (IDEM).

O nível de concentrado da dieta não afetou as estimativas de exigências de energia líquida para mantença. Isto pode ser explicado pela similaridade do tamanho relativo dos órgãos associados à produção de calor corporal e aos gastos de energia com mantença, como pulmão, fígado, coração e vísceras, observada entre os tratamentos (Morais et al., 2014). De acordo com o Nutrient... (2007), aproximadamente $50 \%$ da energia ingerida pelo animal é utilizada para manutenção desses tecidos.
O intercepto da Eq. [5], representado na Fig. 1, indica que, quando a ingestão diária de energia metabolizável for zero, o valor estimado para produção de calor é $57 \mathrm{kcal} / \mathrm{PCJ}^{0,75} /$ dia. Este representa a exigência de energia líquida para mantença (ELm).

$\mathrm{Y}=0,488 * \mathrm{X}+0,057$

Eq. [5],

em que: "Y" é a produção de calor $\left(\mathrm{Mcal} / \mathrm{PCJ}^{0,75}\right)$; e " $\mathrm{X}$ " é a ingestão diária de energia metabolizável (Mcal/kg PCJ ${ }^{0,75}$ ). 
Esse resultado, obtido pela análise dos dados em conjunto, pode ser assumido como a exigência líquida de mantença (ELm) de borregas em crescimento, independentemente do nível de concentrado na dieta. Esse valor está próximo aos valores descritos pelo Nutrient... (1980) e pelo Nutrient... (2007), 58,6 e $56 \mathrm{kcal} / \mathrm{kg} \mathrm{PCJ} \mathrm{PJ}^{0,75}$, respectivamente. Cabral et al. (2008), ao revisarem os dados brasileiros, sugeriram para estimativa das exigências de energia líquida de mantença de ovinos valor similar ao observado neste trabalho $\left(57 \mathrm{kcal} / \mathrm{kg} \mathrm{PCJ} \mathrm{J}^{0,75} / \mathrm{dia}\right)$.

Houve aumento das exigências de EMm com a inclusão dos níveis crescentes de concentrado na dieta (Tab. 2).

Tabela 2. Parâmetros e erros-padrão dos parâmetros das equações de predição da energia retida (ER) em função da ingestão diária de energia metabolizável (IDEM) e exigências de energia metabolizável para mantença $(\mathrm{EMm})$ em cada nível de concentrado

\begin{tabular}{|c|c|c|c|}
\hline \multirow{2}{*}{$\begin{array}{c}\text { Nível de concentrado na } \\
\text { dieta }(\%)\end{array}$} & \multicolumn{2}{|c|}{ Parâmetros da equação ${ }^{1}$} & \multirow{2}{*}{$\begin{array}{l}\text { Exigência de energia metabolizável } \\
\text { para mantença }{ }^{2}, \mathrm{Mcal} / \mathrm{kg} \mathrm{PCJ}^{0,75}\end{array}$} \\
\hline & A & $\mathrm{b}$ & \\
\hline 20 & $-0,0285 \pm 5,39 \times 10^{-3}$ & $0,3040 \pm 3,05 \times 10^{-2}$ & 0,094 \\
\hline 40 & $-0,0392 \pm 7,17 \times 10^{-3}$ & $0,4056 \pm 3,77 \times 10^{-2}$ & 0,097 \\
\hline 60 & $-0,0470 \pm 6,36 \times 10^{-3}$ & $0,4743 \pm 3,10 \times 10^{-2}$ & 0,099 \\
\hline 80 & $-0,0598 \pm 5,56 \times 10^{-3}$ & $0,5827 \pm 2,70 \times 10^{-2}$ & 0,103 \\
\hline
\end{tabular}

${ }^{1} \mathrm{ER}=\mathrm{a}+\mathrm{b} \times$ IDEM; em que ER é a energia retida no corpo por dia (kcal/PCVZ ${ }^{0,75}$ ), "a" e "b" são parâmetros da equação, e IDEM é a ingestão diária de energia metabolizável $\left(\mathrm{Mcal} / \mathrm{kgPVJ}^{0,75}\right)$.

${ }^{2}$ Estimado como o valor de IDEM quando a ER se igualou a zero.

Esse resultado pode ser associado ao fato de que a inclusão de concentrado na dieta proporcionou aumento no ganho de peso dos animais e, consequentemente, diferenças no peso corporal final entre os tratamentos (Ribeiro, 2011). Segundo o Nutrient... (2007), os animais consomem para atender o máximo potencial de crescimento e, dessa forma, quanto maior o animal, maior a ingestão de energia necessária para isso. Assim, as exigências de energia metabolizável de mantença foram crescentes para possibilitar a manutenção dos processos fisiológicos associados ao metabolismo intermediário e visceral bem como a síntese e a deposição de tecidos, especialmente de músculo e gordura na carcaça.

Com o aumento das exigências de EMm, houve uma redução linear da eficiência de uso da energia metabolizável para mantença $(\mathrm{km})$, pois a Elm foi constante para os animais, independentemente do nível de concentrado (Eq. [6] e Fig. 2). $\mathrm{km}=0,6252-0,0009 \times$ nível de concentrado $(\mathrm{C})$ Eq. [6].

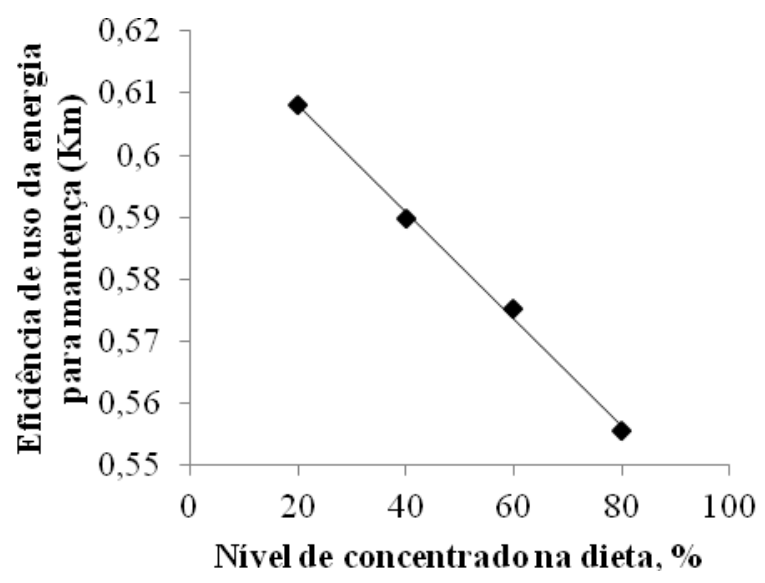

Figura 2. Variação da eficiência de uso da energia metabolizável para mantença $(\mathrm{km})$ em função do nível de concentrado na dieta. 
Essa redução da eficiência de uso da energia metabolizável para mantença poderia ser explicada pela menor eficiência na absorção e na utilização de metabólitos à medida que houve aumento do ganho de peso dos animais. No entanto, considerando que as borregas de todos os tratamentos apresentaram o consumo de matéria seca similar (Ribeiro, 2011) e o valor de NDT se estabilizou a partir da inclusão de $60 \%$ de concentrado na dieta (Tab. 1), infere-se que os menores níveis de eficiência de uso de energia não estão associados a processos digestivos, mas ao aproveitamento dos nutrientes após a absorção.

Ferrell e Jenkins (1985) também afirmaram que quanto melhor o nível nutricional dos animais, menor a eficiência dos animais na utilização da energia para mantença. Esses autores associaram essa diferença à plasticidade da mantença em animais com alimentação restrita. Nesse caso, quanto menor o nível nutricional dos animais, melhor a eficiência de utilização da energia metabolizável para mantença devido à capacidade do organismo do animal de reduzir o tamanho e a taxa metabólica de vários órgãos, tecidos e vísceras.

Os níveis de concentrado não afetaram as estimativas das exigências de energia líquida para ganho. Assim, a ELg pôde ser estimada de acordo com a Eq. [7] independentemente do nível de concentrado na dieta.

$\mathrm{ELg}=0,524 * \mathrm{PCJ}^{0,75} * \mathrm{GPCVZ}^{1,21} \quad$ Eq. [7],

em que: ELg é a energia líquida para ganho de peso (Mcal/dia); PCJ é o peso corporal em jejum (kg); e GPCVZ é o ganho de peso de corpo vazio (kg/dia).

Foi observado efeito linear positivo (Eq. [8], Fig. 3) do nível de concentrado sobre a eficiência do uso da energia metabolizável para ganho $(\mathrm{kg})$.

$$
\begin{aligned}
& \mathrm{kg}=0,2155+0,0045 \times \text { nível de } \\
& \text { concentrado (C) }
\end{aligned}
$$

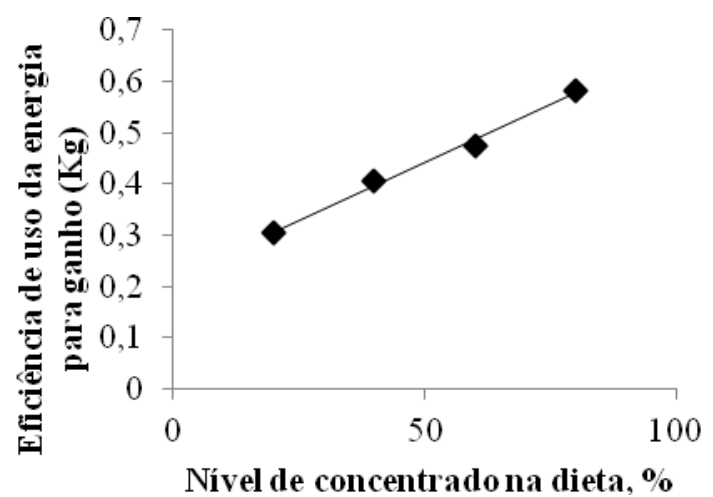

Figura 3. Variação da eficiência de uso da energia metabolizável para ganho em função do nível de concentrado na dieta.

Essa melhoria na eficiência do uso da energia metabolizável para o ganho $(\mathrm{kg})$ com o aumento do nível de concentrado na dieta provavelmente contribuiu para o maior ganho de peso desses animais. Na recria, o consumo de matéria seca (g/dia) e o ganho médio diário (g/dia) foram $1196 \pm 28$ e $135 \pm 14 ; 1139 \pm 23$ e $168 \pm 11 ; 1177 \pm 23$ e $218 \pm 11 ; 1193 \pm 28$ e $246 \pm 13$ para os níveis de $20,40,60$ e $80 \%$ de concentrado, respectivamente. Já na terminação, foram observados $1450 \pm 41$ e $162 \pm 19 ; 1328 \pm 31$ e $159 \pm 15 ; 1295 \pm 31$ e $162 \pm 15 ; 1207 \pm 41$ e $171 \pm 19$ g/dia (Ribeiro, 2011).
Embora as exigências líquidas de ganho (ELg) não tenham sido afetadas pelos níveis crescentes de concentrado, provavelmente a relação músculo:gordura da carcaça das borregas foi distinta entre os tratamentos. Nesse contexto, os maiores valores de $\mathrm{kg}$ com a inclusão dos níveis crescentes de concentrado na dieta podem estar associados ao aumento da deposição de tecido adiposo em resposta ao ganho de peso proporcionado pela maior ingestão de energia. Segundo Owens et al. (1995), quanto maior a proporção de gordura no ganho, maior será o kg, pois existem diferenças de eficiência de utilização da energia metabolizável para 
deposição de tecidos muscular e adiposo. Para cada grama de proteína são depositadas $5,6 \mathrm{kcal}$, enquanto $9,4 \mathrm{kcal}$ são retidas em cada grama de lipídio depositado. Assim, conforme o animal se aproxima do tamanho à maturidade, mesmo recebendo dietas com mesmo teor de energia nas fases de crescimento e terminação, aproveita melhor cada kcal fornecida pela dieta para deposição de gordura.

Considerando-se a estimativa do valor de exigência líquida de mantença (Eq. [5]) e as exigências líquidas de ganho (Eq. [7]), foi possível obter os respectivos valores para cada categoria de peso corporal (Tab. 3) e comparálos aos propostos pelo Nutrient... (2007). Os valores de exigências de energia líquida de mantença estimados neste trabalho foram próximos aos valores recomendados pelo Nutrient... (2007) e apresentaram menos de 2\% de variação em relação às predições desse conselho.

Tabela 3. Exigências de energia líquida para mantença (ELm, Mcal/dia) e energia líquida para ganho (ELg, Mcal/dia) para borregas de diferentes pesos vivos, utilizando-se as equações de predição obtidas por meio dos dados experimentais e das equações propostas pelo Nutrient... (2007)

\begin{tabular}{ccccc}
\hline \multirow{2}{*}{ Peso corporal $(\mathrm{kg})$} & \multicolumn{2}{c}{ Equações preditas } & \multicolumn{2}{c}{ NRC (2007) } \\
\cline { 2 - 5 } & ELm (Mcal/dia) & ELg (Mcal /dia) & ELm (Mcal/dia) & ELg (Mcal/dia) \\
\hline 20 & 0,821 & 0,465 & 0,806 & 0,360 \\
30 & 1,231 & 0,698 & 1,209 & 0,488 \\
40 & 1,642 & 0,930 & 1,613 & 0,606 \\
\hline
\end{tabular}

Por outro lado, ao se compararem os valores de exigência de ELg (Mcal/dia), observa-se que os valores obtidos no presente trabalho foram superiores $(40 \%$ em média) aos recomendados pelo Nutrient... (2007). Essa diferença pode estar associada ao efeito de raça, pois os animais lanados, utilizados no presente trabalho, são oriundos de cruzamento com a raça Pantaneira, enquanto os animais usados na elaboração das recomendações do Nutrient... (2007) são provenientes de raças com melhor potencial genético para ganho e/ou maior tamanho à maturidade, fato que implica composição do ganho mais magra, refletindo em menor ELg. Assim, a maior ELg estimada pelas equações de predição deste trabalho indica que as borregas depositaram gordura corporal precocemente quando receberam maiores proporções de concentrado.

Utilizando-se as equações apresentadas anteriormente para estimativa das eficiências de uso da EM, foram calculadas as exigências diárias de EMm, EMg e EM total para borregas com 20,30 e 40kg de peso vivo recebendo níveis crescentes de concentrado. Adicionando-se
$21,9 \%$ aos valores de EM $(\mathrm{ED}=\mathrm{EM} / 0,82)$ para estimar a ED e considerando-se que cada quilograma de NDT equivale a 4,409Mcal de $\mathrm{ED}$, foram estimadas as exigências de NDT dos animais (Tab. 4).

As exigências de EMm e EMg foram influenciadas pelos níveis de concentrado das dietas. $\mathrm{O}$ aumento das exigências de EMm com a inclusão dos níveis crescentes de concentrado possivelmente é resultado da demanda de energia necessária para manter as atividades fisiológicas do organismo em função da elevação do peso corporal das borregas, já que os animais consomem conforme crescem (Nutrient..., 2007).

Por outro lado, os valores de exigências de EMg reduziram-se com o aumento da proporção de concentrado na dieta. Esse resultado era esperado, pois, nesse caso, os valores decrescentes das exigências de EMg são devido ao aumento da eficiência do uso da energia para ganho de peso (Fig. 3) associados aos valores similares de ELg, variáveis utilizadas em seu cálculo $(\mathrm{ELg} / \mathrm{kg})$. 
Exigências de energia...

Tabela 4. Exigências de energia metabolizável para mantença (EMm), para ganho (EMg), energia metabolizável total diária (EM diária) e exigência diária de nutrientes digestíveis totais (NDT) de borregas recebendo dietas com diferentes níveis de concentrado

\begin{tabular}{|c|c|c|c|c|}
\hline Peso vivo $(\mathrm{kg})$ & EMm (Mcal/dia) & EMg (Mcal/dia) & EM diária (Mcal/dia) & NDT (kg/dia) \\
\hline \multicolumn{5}{|c|}{$20 \%$ de concentrado } \\
\hline 20 & 1,352 & 1,523 & 2,875 & 0,795 \\
\hline 30 & 2,028 & 2,284 & 4,312 & 1,193 \\
\hline 40 & 2,704 & 3,046 & 5,749 & 1,590 \\
\hline \multicolumn{5}{|c|}{$40 \%$ de concentrado } \\
\hline 20 & 1,393 & 1,176 & 2,569 & 0,711 \\
\hline 30 & 2,090 & 1,765 & 3,854 & 1,066 \\
\hline 40 & 2,786 & 2,353 & 5,139 & 1,421 \\
\hline \multicolumn{5}{|c|}{$60 \%$ de concentrado } \\
\hline 20 & 1,437 & 0,958 & 2,395 & 0,663 \\
\hline 30 & 2,155 & 1,437 & 3,593 & 0,994 \\
\hline 40 & 2,874 & 1,917 & 4,791 & 1,325 \\
\hline \multicolumn{5}{|c|}{$80 \%$ de concentrado } \\
\hline 20 & 1,484 & 0,808 & 2,292 & 0,634 \\
\hline 30 & 2,226 & 1,213 & 3,438 & 0,951 \\
\hline 40 & 2,967 & 1,617 & 4,584 & 1,268 \\
\hline
\end{tabular}

As exigências de EM diária total, assim como as exigências em NDT estimadas, foram maiores que os valores preconizados pelo Nutrient... (2007). Ambas apresentaram comportamento similar, pois as exigências de NDT foram obtidas das EM multiplicadas por um fator fixo. Ao se avaliarem as exigências de EM diária preditas pelo Nutrient... (2007), é possível observar valores de 2,100; 3,008 e 3,926Mcal/dia para animais de 20,30 e $40 \mathrm{~kg}$, respectivamente, independentemente do nível de concentrado na dieta, sendo estes $24,85 \%$ menores em relação aos obtidos no presente trabalho. Esses resultados distintos são reflexos das diferenças na eficiência de utilização da energia metabolizável para os processos de mantença e ganho, que, segundo esse comitê, pode variar principalmente em função da raça, do sexo, da idade e do manejo alimentar dos animais.

\section{CONCLUSÕES}

A exigência líquida para mantença de borregas mestiças lanadas pode ser estimada pela equação: $\operatorname{ELm}(\mathrm{Mcal} / \mathrm{dia})=0,057 * \mathrm{PCJ}^{0,75}$. A exigência líquida de energia para ganho de peso de borregas mestiças pode ser estimada pela equação: $\operatorname{ELg}(\mathrm{Mcal} / \mathrm{dia})=0,524 \times \mathrm{PVJ}^{0,75} \mathrm{x}$ GPCVZ $^{1,21}$. De acordo com o nível de concentrado (C) utilizado nas dietas, recomendase a equação $\mathrm{km}=0,6252-0,0009 \times \mathrm{C}$ para estimativa da eficiência de uso da energia metabolizável para mantença e $\mathrm{kg}=$ $0,2155+0,0045 \times \mathrm{C}$ para estimativa da eficiência de uso da energia metabolizável para ganho. Os valores de exigências de energia líquida de mantença estimada para borregas mestiças lanadas com 20 a 40kg são próximos aos valores recomendados pelo Nutrient... (2007), no entanto os valores de exigência líquida para ganho de peso são superiores.

\section{REFERÊNCIAS}

BURNHAM, K.P.; ANDERSON, D.R. Model selection and multimodel inference: a practical information-theoretic approach. 2.ed. New York: Springer, 2002. 488p.

CABRAL, L.S.; NEVES, E.M.O.; ZERVOUDAKIS, J.T. et al. Estimativas dos requisitos nutricionais de ovinos em condições brasileiras. Rev. Bras. Saúde Prod. Anim. v.9, p.529-542, 2008.

FERRELL, C.L.; JENKINS, T.G. Cow type and the nutritional environment: nutritional aspects. J. Anim. Sci., v.61, p.725-741, 1985.

HALL, M.B. Calculation of non-structural carbohydrate content of feeds that contain nonprotein nitrogen. Florida: University of Florida, 2000. p.A25-A34. (Bulletin 339). 
MORAIS, M.G.; SILVA, R.A.; COMPARIN, M.A.S. et al. Carcass and non-carcass components of feedlot ewes subjected to increasing levels of concentrate in the diet. Rev. Bras. Saúde Prod. Anim., v.15, p.1038-1051, 2014.

NUTRIENT requirements of farm animals. London: Landmark, 1980. 351p.

NUTRIENT requirements of small ruminants. Washington: National Academy Press, 2007. 362 p.

OFFICIAL methods of analysis. 15.ed. Virginia: AOAC, 1990.

OWENS, F.N.; GILL，D.R.; SECRIST, D.S; COLEMAN, S.W. Review of some aspects of growth and development of feedlot cattle. J. Anim. Sci., v.73, p.3152-3172, 1995.

REGAZZI, A.J. Teste para verificar a igualdade de parâmetros e a identidade de modelos de regressão não-linear. Rev. Ceres, v.50, p.9-26, 2003.
RIBEIRO, C.B. Exigência proteica de fêmeas ovinas mestiças. 2011. 77f. Dissertação (Mestrado em Ciência Animal) - Faculdade de Medicina Veterinária e Zootecnia, Universidade Federal de Mato Grosso do Sul, Campo Grande, MS.

SAS. Statistical analysis system. Versão 9.2. Cary: SAS Institute, 2009.

TEDESCHI, L.O. Model evaluation system MES, 2006. Disponível em: $<$ http.//nutritionmodels.tamu.edu/mes.htm $>$. Acessado em: 5 nov. 2010.

WALLACH, D.; GOFFINET, B. Mean squared error of prediction as a criterion for evaluating and comparing system models. Ecol. Model., v.44, p.299-306, 1989. 